\title{
AN INTELLIGENT TUTORING SYSTEM FOR OPERATORS' TRAINING IN POWER SYSTEM CONTROL CENTRES
}

\author{
Luiz Faria, António Silva, Zita Vale, Carlos Ramos \\ Knowledge Engineering and Decision Support Research Centre, Institute of Engineering - Polytechnic of Porto, Portugal \\ lff@dei.isep.ipp.pt, asilva@dei.isep.ipp.pt,zav@isep.ipp.pt, csr@dei.isep.ipp.pt \\ Albino Marques \\ REN - Energy Networks of Portugal, Porto, Portugal \\ albino.marques@ren.pt
}

Keywords: Intelligent Tutoring Systems, Intelligent Alarm Processing, Diagnosis, Restoration, Control Centres, Power Systems.

\begin{abstract}
The activity of Control Center operators is important to guarantee the effective performance of Power Systems. Operators' actions are crucial to deal with incidents, especially severe faults, like blackouts. In this paper we present an Intelligent Tutoring approach for training Portuguese Control Centre operators in tasks like incident analysis and diagnosis, and service restoration of Power Systems. Intelligent Tutoring System (ITS) approach is used in the training of the operators, taking into account context awareness and the unobtrusive integration in the working environment.
\end{abstract}

\section{INTRODUCTION}

Current Power Systems are highly complex and require sophisticated and precise operation and control. The most important real-time decisions concerning Power System operation are taken in Control Centres where human operators are the final link of a complex chain. Although Power System reliability has been increasing, incidents with more or less severe consequences still occur. In some cases, this can result in blackout situations, leading to consumer lack of supply, for which the economic and social impact can be dramatically high. Figure 1 shows the impact of the 14th August 2003 blackout in the Northeast part of USA.

Blackouts have been a major concern in Power Systems mainly since the occurrence of the 9th November 1965 Northeast Blackout in USA. In recent years, several blackouts caused major concerns making the need to keep lights on more important than ever. IEEE Power \& Energy magazine edited a special issue titled "Shedding light on blackouts - From prevention through restoration" (vol.4, no. 5, September/October 2006). On the 4th October 2006, a Saturday, some minutes after 10 p.m., the UCTE (Union for the Coordination of Transmission of Electricity) European Network experienced a quasi blackout situation affecting 9 European countries and North Africa and about 10 million consumers, originated from a switching-off of the $380 \mathrm{kV}$ double circuit line Conneforde-Diele in Germany.
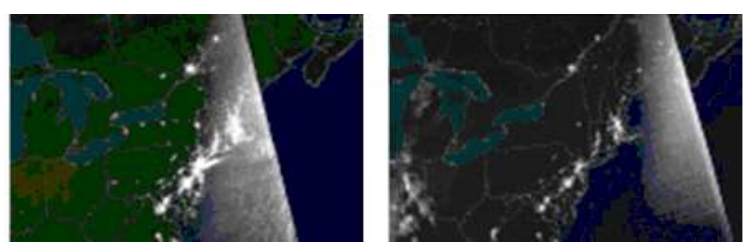

Figure 1: Northeast USA before and after the 14th August 2003 Blackout (Source: NOAA - National Oceanic \& Atmospheric Administration)

Control Center operators' performance is determinant to minimize the incident consequences. The need of a good response of Control Centres to severe faults, like blackouts, is even more important nowadays, due to the generalization of the Electricity Markets (Praça, Ramos, Vale, and Cordeiro 2003). As Power Systems reliability 
increased, the number of incidents offering occasion for operator on-the-job training has decreased. The consequences of incorrect operator behaviour are all more severe during a serious incident (Vale et all 1997). Operator training is vital for overcoming these problems, as well as the availability of decision support tools.

Intelligent Tutoring Systems (ITS) has been the main approach selected to deal with the operators' training in diagnosis and restoration tasks, namely because:

1. They represent domain knowledge in a structured way, allowing the inference of new knowledge (access to the essential knowledge).

2. They model the trainee, allowing action in a non-monotonous way, adapting better to the trainee's characteristics and evolution (awareness of the needs of people).

3. With the right didactic knowledge they allow the system to choose different pedagogical approaches in the different phases of the learning process (requirements customization).

4. They are able to constantly monitor the trainee's performance and evolution, gathering information to guide the system's adaptation (context awareness).

5. They typically require very little intervention from the training staff, and can be used in the working environment without disturbing the normal working routines (unobtrusivity).

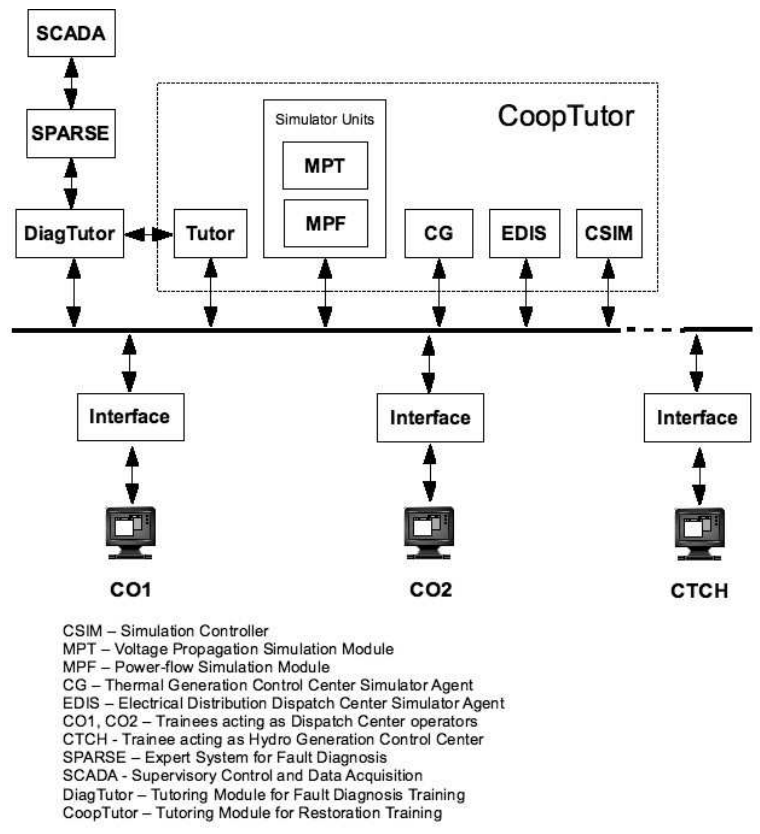

Figure 2: Tutoring Environment Architecture
In this paper we present an Intelligent Tutoring System used for training Control Centre operators in fault diagnosis and power restoration. Several Artificial Intelligence techniques are used to make this system able to minimize network experts need in training preparation and to enable on the job and cooperative effective training.

The Intelligent Tutoring System that has been developed for the Control Centre operators involves two main areas: one devoted to the training of fault diagnosis skills and another dedicated to the training of power system restoration techniques. Figure 2 shows this tutoring environment architecture.

\section{TUTORING MODULE FOR FAULT DIAGNOSIS TRAINING}

In order to illustrate how a training session is conducted and the interaction between the operator and the tutor, this section presents a very simplified diagnosis problem containing a DmR (monophase tripping with reclosure) incident, occurred in panel 204 of Ermesinde substation (SED). The relevant SCADA messages related to this incident are depicted in Table 1. These SCADA messages correspond to the following events: breaker tripping, breaker moving and breaker closing (Vale et all 1997). In a real training scenario the operator is faced with a huge amount of messages, typically several hundreds.

Table 1: Incident in panel 204 of SED substation.

\begin{tabular}{|ll|l|l|l|ll|ll|}
\hline $14-D E C-2003$ & $04: 24: 45.200$ & SED & 204 & CCL, 2 & >> TRIPPING & 0 & 1 \\
\hline $14-D E C-2003$ & $04: 24: 45.240$ & SED & 204 & CCL, 2 & -BK & BREAKER & 0 & 0 \\
\hline $14-D E C-2003$ & $04: 24: 45.860$ & SED & 204 & CCL, 2 & -BK & BREAKER & 0 & 1 \\
\hline
\end{tabular}

\subsection{Reasoning About Operator Answers}

The interaction between the trainee and the tutor is performed through prediction tables (Figure 3) where the operator selects a set of premises and the corresponding conclusion. The premises represent events (SCADA messages), temporal constraints between events or previous conclusions (Faria, Vale, and Ramos, 2005). 


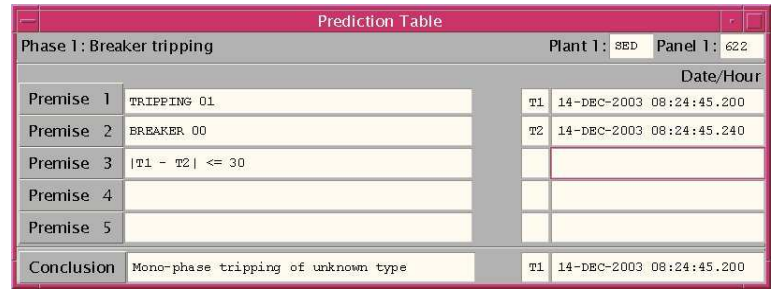

Figure 3: Prediction Table

DiagTutor does not require the operator's reasoning to follow a predefined set of steps, as in other implementations of the model tracing technique (Anderson and all, 1995). In order to evaluate this reasoning, the tutor will compare the prediction tables' content with the specific situation model. This model is obtained by matching the domain model with the inference undertaken by SPARSE expert system (Vale et all, 1997). This process is used to: identify the errors revealing operator's misconceptions; provide assistance on each problem solving action, if needed; monitor the trainee knowledge evolution; and provide learning opportunities for the trainee to reach mastery.

The identified errors are used as opportunities to correct the faults in the operator's reasoning. The operator's entries in prediction tables cause immediate responses from the tutor. In case of error, the operator can ask for help which is supplied as hints. Hinting is a tactic that encourages active thinking structured within guidelines dictated by the tutor. The first hints are generic, becoming more detailed if the help requests are repeated.

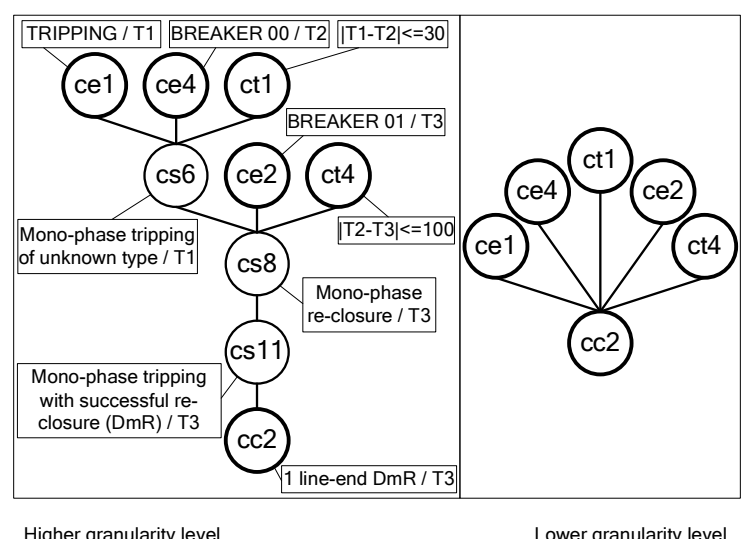

Figure 4: Higher and lower granularity levels of the situation specific model

The situation specific model generated by the tutoring system for the problem presented is shown in the left frame of Figure 4. It presents high granularity since it includes all the elementary steps used to get the problem solution. The tutor uses this model to detect errors in the operator reasoning by comparing the situation specific model with the set of steps used by the operator. This model's granularity level is adequate to a novice trainee but not to an expert operator. The right frame of Figure 4 represents a model used by an expert operator, including only concepts representing events $\left(\mathrm{ce}_{\mathrm{i}}\right)$, temporal constraints between events $\left(\mathrm{ct}_{\mathrm{i}}\right)$ and the intermediate $\left(\mathrm{cs}_{\mathrm{i}}\right)$ and final conclusions $\left(\mathrm{cc}_{\mathrm{i}}\right)$. Any reasoning model between the higher and lower granularity level models is admissible since it does not include any violation to the domain model. These two levels are used as boundaries of a continuous cognitive space.

\subsection{Adapting the Curriculum to the Operator}

The main goal of the Curriculum Planning module is to select, from a library, a problem fitting the trainee needs.

The preparation of the tutoring sessions' learning material is a time-consuming task. In the industrial environment, there is not usually a staff exclusively dedicated to training tasks. Specifically, in the electrical sector, the preparation of training sessions is done by the most experienced operators which are often overloaded with power system operation tasks (Faria, Vale and Ramos 2000). In order to overcome this difficulty, we developed two tools. The first one generates and classifies training scenarios from real cases previously stored. As these may not cover all the situations that control centre operators must be prepared to face, another tool is used to create new training scenarios or to edit already existing ones (Faria, Vale and Ramos 2000).

The process used by the Curriculum Planning module to define the problems' features involves two phases. First, the tutor must define the difficulty level of the problem, using heuristic rules. These rules relate parameters like the trainee's performance in previous problems and his overall level of knowledge. In the second phase, the tutor uses the user model's contents to choose the type of the most suitable incidents to be included in the problem, taking into account the domain concepts involved in each type of incident and the corresponding trainee's expertise. 


\subsection{Difficulty Level Selection}

To evaluate the problems' difficulty level, we need to identify the cases' characteristics that increase their complexity, namely by the: number of incidents involved in the case; variety of incident types; number of involved plants; and existence of chronological inversion in SCADA messages.

The choice of the difficulty level depends on two factors contained in the trainee's model: the trainee's global knowledge and a global acquisition factor. The first parameter is a measure of the trainee' knowledge level in the whole range of domain concepts and is calculated using the mean of his knowledge level in each domain concept. The Curriculum Planning Module needs appropriate thresholds for deciding on the next problem difficulty level. The opinion of the trainees, regarding their personal evolution as the problems difficulty level is changed, can be used to tune these thresholds.

The acquisition factors record how well trainees learn new concepts. When a new concept is introduced, the tutor monitors the trainee performance on the first few problems, namely how well and how quickly he solves them. This analysis determines the trainee's acquisition factor. The procedure used to determine the trainee's acquisition in each domain concept is based on the number of times the trainee's knowledge level about the concept increased, considering the three first applications of the concept.

The mechanism used to define the difficulty level of the problems is based on the following rule:

If the global knowledge level and the global acquisition factor change in opposite directions Then the problem difficulty level does not change Else the problem difficulty level changes in the same direction of the global knowledge level.

\subsection{Problem Type Adequacy to the Trainee Cognitive Status}

The mechanism used to classify each kind of incident in terms of adequacy to the trainee is based on a neural network (Fig. 5). The nodes belonging to the input layer correspond to the concepts included in the domain's knowledge base (to be assimilated by the trainees). Each node represents the application of a concept in a specific context. For instance, the nodes ce1/T1 and ce1/T5 represents two instances of the same concept and characterize the application of the concept of breaker tripping in the situations of first tripping and tripping after an automatic reclosure. The input vector contains an estimate of the trainee's expertise level for each concept or its application and is obtained from the user model. Therefore, this vector represents an estimate of the trainee's domain knowledge.

The output layer units represent the adequacy of an incident type to the current learner's knowledge status. The number of units corresponds to the number of incident types of the following types: DS (single tripping); DtR (three-phase tripping with reclosure); DmR (monophase tripping with reclosure); DtD (three-phase tripping with failed reclosure); and $\mathrm{DmD}$ (monophase tripping with failed reclosure). Each output layer's node, representing a type of incident, is connected only to the input nodes corresponding to concepts involved with that incident type. These connections are done with links of weight wij.

The values used as weights are $w i j=\{1,0,-\}$ where ' - ' is used to indicate that there is no connection between node $i$ of the output layer and the input node $\mathrm{j}$. This means that concept $\mathrm{j}$ is not involved in an incident type $i$.

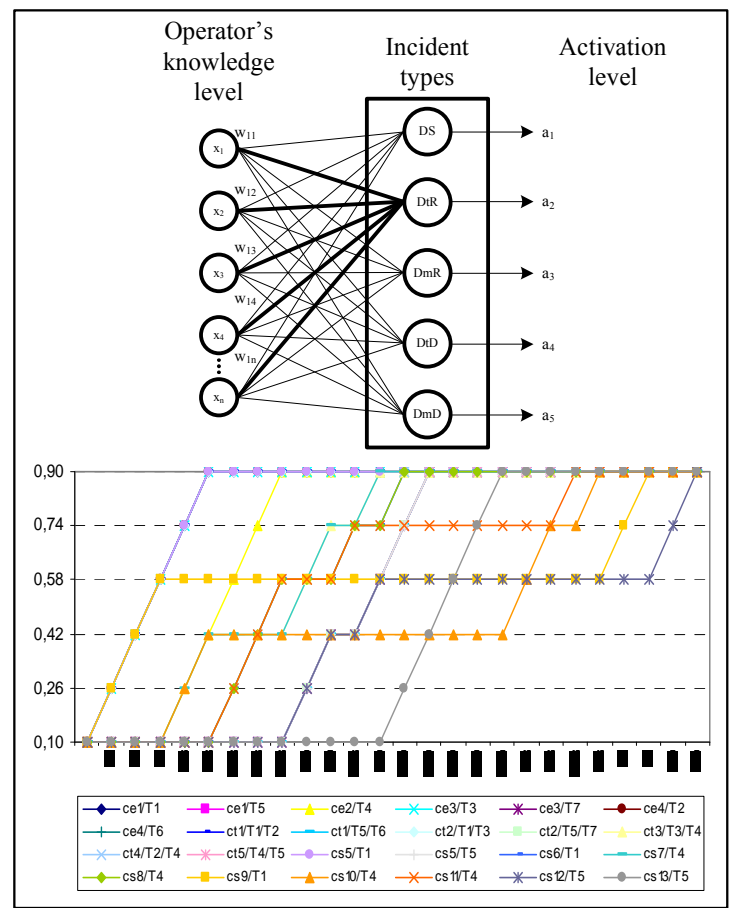

Figure 5: Classification mechanism 
Each output neuron activation level is computed using the input vector and its weight vector. The activation is defined by the Euclidean distance, given by (1).

$$
a_{i}=\sqrt{\sum_{j=1}^{n}\left(w_{i j}-x_{j}\right)^{2}}
$$

We can see that a neuron with a weight vector (w) similar to the activation level vector of the input nodes (x) will have a low activation level and vice versa. The output layer's node with the lowest activation will be the winner.

In Figure 5 we illustrate a situation where all the model variables are set to their minimum value $(0.1)$ and achieve a maximum value of 0.9 . It is also assumed that the ideal operator applies correctly all the domain concepts involved in the problem and that the updating rate is constant.

It can be observed that, after the third iteration, the concepts used in DS incident type overcome the medium level (0.5), leading to a new type of incident (DtR) in the next iteration. After the fourth iteration, some concepts that are not used in DS but are involved in DtR incident overtake the minimum level for the first time.

We observed that an early introduction of new concepts can contribute to increase the instructional process efficiency. The problem selection mechanism ensures that the problem sequence is not monotonous, tending to stimulate the operator's performance with new kinds of incidents.

\section{TUTORING MODULE FOR RESTORATION TRAINING}

\subsection{Restoration Training Issues}

The management of a power system involves several distinct entities, responsible for different parts of the network. The power system restoration needs a close coordination between generation, transmission and distribution personnel and their actions should be based on a careful planning and guided by adequate strategies (Sforna and Bertanza, 2002).

In the specific case of the Portuguese transmission network, four main entities can be identified: the National Dispatch Centre (CC); the Operational Centre (CO); the Hydroelectric Control
Centres (CTCH); and the Distribution Dispatch (EDIS).

The power restoration process is conducted by these entities in such a way that the parts of the grid they are responsible for will be slowly led to their normal state, by performing the actions specified in detailed operating procedures and fulfilling the requirements defined in previously established protocols. This process requires frequent negotiation between entities, agreement on common goals, and synchronization.

The purpose of the training tutor is to allow the training of the established restoration procedures and the drilling of some basic techniques. Power system utilities have built detailed plans containing the actions to execute and the procedures to follow in case of incident. In the case of the Portuguese network, there are specific plans for the system restoration following several cases of sectorial blackouts as well as national blackouts, with or without loss of interconnection with the Spanish network. Table 2 illustrates a service restoration plan.

Table 2: Restoration Plan example.

\begin{tabular}{|c|c|}
\hline $\begin{array}{l}\text { Step } \\
\text { No. }\end{array}$ & PLAN STEP \\
\hline 0 & $\begin{array}{l}\text { Notify Distribution Dispatch Center about the } \\
\text { incident and expected restoration time. Wait for } \\
150 \mathrm{kV} \text { to be available in SRA bus }\end{array}$ \\
\hline 1 & $\begin{array}{l}\text { Feed the } 150 \mathrm{kV} \text { to SRA bus using } 400 / 150 \mathrm{kV} \\
\text { autotransformers }\end{array}$ \\
\hline 2 & Switch SVI substation to manual \\
\hline 3 & $\begin{array}{l}\text { Energize the lines fed by the } 150 \mathrm{kV} \text { bus of } \\
\text { SRA with priority to lines connected to } \\
\text { substations SOR and SRU and to power plants } \\
\text { CCD and CVN }\end{array}$ \\
\hline 4 & $\begin{array}{l}\text { Contact the Hydroelectric Power Plants' CC, } \\
\text { asking for the restoration of their lines with } \\
\text { priority for the ones between CCD and CAR } \\
\text { and between CCD and SVI/CVF }\end{array}$ \\
\hline 5 & $\begin{array}{l}\text { Wait for the automatic operators of SCV and } \\
\text { SGR substations to restore the } 150 / 60 \mathrm{kV} \\
\text { transformers, if no voltage is available in } 60 \mathrm{kV} \\
\text { buses }\end{array}$ \\
\hline 6 & $\begin{array}{l}\text { Wait for SOR substation automatic operator to } \\
\text { restore the service, including the line to SVI }\end{array}$ \\
\hline 7 & $\begin{array}{l}\text { Finish the restoration of } 150 \mathrm{kV} \text { line between } \\
\text { substations SRA and SED }\end{array}$ \\
\hline 8 & $\begin{array}{l}\text { Check if the automatic operators' work is } \\
\text { concluded and finish the restoration if it has not } \\
\text { been done automatically }\end{array}$ \\
\hline 9 & $\begin{array}{l}\text { Notify Distribution Dispatch Center about the } \\
\text { end of the restoration process }\end{array}$ \\
\hline
\end{tabular}


Our Restoration training system use the concept of agents (Jennings and Wooldridge, 1995), that can be seen as virtual entities that possess knowledge about the domain. As real operators, they have tasks assigned to them, goals to be achieved and beliefs about the network status and others agents' activity. They work asynchronously, performing their duties simultaneously and synchronizing their activities only when this need arises. Therefore, the system needs a facilitator (simulator in Figure 2) that supervises the process, ensuring that the simulation is coherent and convincing.

In our system, the trainee can choose to play any of the available roles, namely the $\mathrm{CO}$ and the $\mathrm{CC}$ ones, leaving to the tutor the responsibility of simulating the other participants.

\subsection{Trainee's Model}

The representation method used to model the trainee's knowledge about the domain knowledge is a variation of the Constraint-Based Modelling (CBM) technique (Ohlsson, 1993). This student model representation technique is based on the assumption that diagnostic information is not extracted from the sequence of student's actions but rather from the situation, also described as problem state, that the student arrived at. Hence, the student model should not represent the student's actions but the effects of these actions. Because the space of false knowledge is much greater than the one for the correct one, it was suggested the use of an abstraction mechanism based on constraints. In this representation, a state constraint is an ordered pair (Cr,Cs) where $\mathrm{Cr}$ stands for relevance condition, and $\mathrm{Cs}$ for satisfaction condition. $\mathrm{Cr}$ identifies the class of problem states in which this condition is relevant and Cs identifies the class of relevant states that satisfy Cs. Under these assumptions, domain knowledge can be represented as a set of state constraints. Any correct solution for a problem cannot violate any of the constraints. A violation indicates incomplete or incorrect knowledge and constitutes the basic piece of information that allows the Student Model to be built on.

This CBM technique does not require an expert module and is computationally undemanding because it reduces student modelling processing to a basic pattern matching mechanism. One example of a state constraint can be found below:

If there is a request to CTCH to restore the lines under its responsibility
Then the lines that connect to the hydroelectric power plants must already have been restored Otherwise an error has occurred

Each violation to a state constraint like the one above enables the tutor to intervene both immediately or at a later stage, depending on the seriousness of the error or the pedagogical approach that was chosen.

This technique has allowed us to give the tutor the flexibility needed to address trainees with a wide range of experience and knowledge, tailoring, in a much finer way, the degree and type of support given, and, at the same time, spared us the exhaustive monitoring and interpretation of student's errors during an extended period, which would be required by alternative methods.

Nevertheless, it was found the need for a metaknowledge layer in order to adapt the CBM method to an essentially procedural, time-dependent domain like the power system restoration field. This layer is composed of rules that control the constraints' application, depending on several issues: the phase of the restoration process in which the trainee is; the constraints previously satisfied; and the set of constraints triggered simultaneously.

These rules establish a dependency network between constraints that can be represented by a graph (Figure 6) (Silva, Vale, and Ramos, 1995). The relationships between constraints expressed by this graph can be of precedence, mutual exclusion or priority.

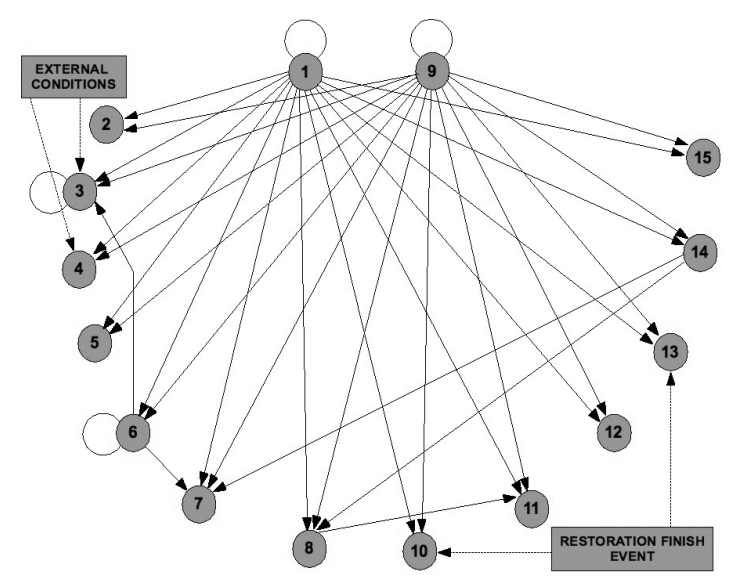

Figure 6: Constraint Dependency Graph 


\subsection{The Cooperative Learning Environment}

This tutor is able to train individual operators as if they were in a team, surrounded by virtual "operators", but is also capable of dealing with the interaction between several trainees engaged in a cooperative process. It provides specialized agents to fulfil the roles of the missing operators and, at the same time, monitors the cooperative work, stepping in when a serious imbalance is detected. The tutor can be used as a distance learning tool, with several operators being trained at different locations.

To support the tutor monitoring activities of the cooperative discussion and decision processes, several provisions were made in order to be able to accurately model the interactions between trainees. The core data contained in the student model has been complemented with information concerning the quantity and characteristics of the interactions detected between trainees. This data is gathered by the tutor by means of a loose monitoring of the interaction patterns coupled with a surface level analysis of the messages contents.

The tutor will be active by its own initiative only if it detects a clear imbalance in the discussion process. It may be called to step in though by the trainees themselves, if they agree on a course of action or if they find themselves in an impasse situation. In the first case, the tutor will use the knowledge contained in the CBM module to evaluate the divergent proposals. In the later case, it will combine the constraint satisfaction data previously gathered with procedural knowledge containing the sequence of the specific restoration plan, in order to issue recommendations about the next step to fulfil.

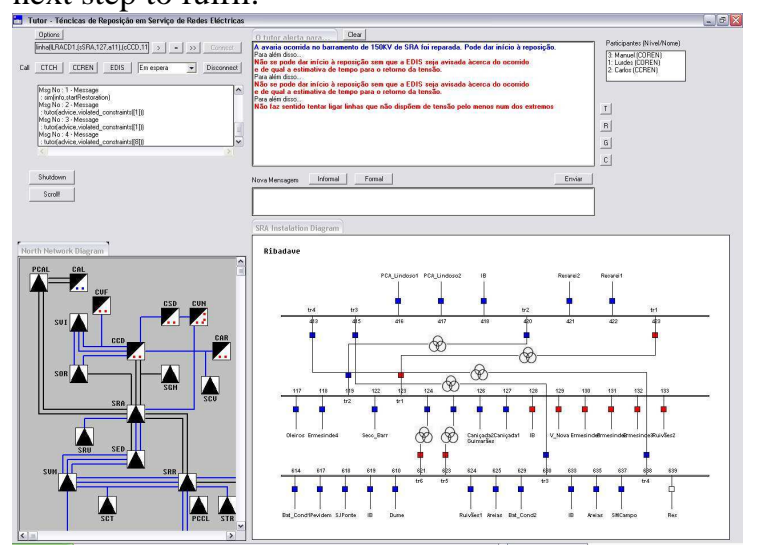

Figure 7: CoopTutor Interface
The general aspect of the ITS interface is shown in Figure 7.

\section{CONCLUSIONS}

This paper described how an Intelligent Tutoring System can be used for the training of Power Systems Control Centre operators in two main tasks: Incident Analysis and Diagnosis; and Service Restoration. Several Artificial Intelligence (AI) techniques were joined to obtain an effective Intelligent Tutoring environment, namely: MultiAgent Systems, Neural Networks, Constraint-based Modelling, Intelligent Planning, Knowledge Representation, Expert Systems, User Modelling, and Intelligent User Interfaces.

The developed system is used in the training of Electrical Engineering BSc students, since the selection of new operators is done from this kind of students. It is also important to notice that this environment has been selected as one of the most important systems combining AI techniques to be available in the "AI-50 years" Exhibition in Portugal (Ramos, 2006), being experimented by many undergraduate students, motivating them for the Electrical Engineering and Computer Science fields.

Concerning the operators' training, the most interesting features of this environment are:

1. The connection with SPARSE, a legacy Expert System used for Intelligent Alarm Processing (Vale et al, 1997).

2. The use of prediction tables and different granularity levels for fault diagnosis training.

3. The use of the model tracing technique to capture the operator's reasoning.

4. The development of two tools to help the adaptation of the curriculum to the operator - one that generates training scenarios from real cases and another that assists in creating new scenarios.

5. The automatic assignment of the difficulty level to the problems.

6. The identification of the operators' knowledge acquisition factors.

7. The automatic selection of the next problem to be presented, using Neural Networks.

8. The use of Multi-Agent Systems paradigm to model the interaction of several operators during system restoration.

9. The use of the Constraint-based Modelling technique in restoration training.

10. The availability of an Intelligent User Interface in the interaction with the operator. 


\section{ACKNOWLEDGEMENTS}

The authors would like to thank FCT foundation and FEDER, PEDIP, POSI, POSC, and PTDC programmes for their support in several research projects leading to the development of the work described here.

\section{REFERENCES}

Anderson, J., Corbett, A., Koedinger, K., and Pelletier, R., "Cognitive Tutors: Lessons Learned", The Journal of the Learning Sciences, v. 4, no. 2, pp. 167-207, 1995

Faria, L., Vale, Z., Ramos, C., Silva, A, and Marques, A., "Training Scenarios Generation Tools for an ITS to Control Center Operators", Proc. Intelligent Tutoring Systems Conf. (ITS'2000).

Faria, L., Vale, Z., Ramos, C., "Diagnostic Tasks Training Based on a Model Tracing Approach", International Journal of Engineering Intelligent Systems for Electrical Engineering \& Communications (CRL), v. 13, no. 4, pp. 223-230, 2005.

Jennings, N., and Wooldridge, M., "Applying agent technology", Applied Artificial Intelligence: An International Journal, Taylor \& Francis London, 9 (4) 1995, 351-361

Ohlsson, S., "Constraint-Based Student Modeling", Student Modeling: the Key to Individualized Knowledge-based Instruction, Greer and McCalla, Editors, Springer-Verlag, 1993, 167-189.

Praça, I., Ramos, C., Vale, Z., and Cordeiro, M., "MASCEM: A Multiagent System That Simulates Competitive Electricity Markets", IEEE Intelligent Systems- Special Issue on Agents and Markets, 18(6), pp. 54-60, 2003.

Ramos, C., "How Portugal Celebrated AI's 50th Anniversary", IEEE Intelligent Systems, 21(4), pp. 8688, 2006.

Sforna, M., Bertanza, V., "Restoration Testing and Training in Italian ISO", IEEE Transactions on Power Systems, November 2002, vol 17, No 4

Silva, A., Vale, Z., Ramos, C., "Cooperative Training of Power Systems Restoration Techniques", 13rd International Conference on Intelligent Systems Applications to Power Systems, Washington, November 2005

Vale, Z., Moura, A., Fernandes, M., Marques, A., Rosado, A. and Ramos, C., "SPARSE: An Intelligent Alarm Processor and Operator Assistant", IEEE ExpertSpecial Track on AI Applications in the Electric Power Industry, 12(3), pp. 86- 93, 1997. 